\title{
Desenvolvimento ponderal e diversidade fenotípica entre cruzamentos de ovinos Dorper com raças locais
}

\author{
Paulo Luiz Souza Carneiro(1), Carlos Henrique Mendes Malhado(1), Antonio Alcione Oliveira de Souza Júnior(2), \\ Antonio Gonçalves Serafim da Silva(2), Fabiana Nery dos Santos ${ }^{(1)}$, Pollianna Ferro Santos ${ }^{(1)}$ \\ e Samuel Rezende Paiva ${ }^{(3)}$
}

\begin{abstract}
(1)Universidade Estadual do Sul da Bahia, Dep. de Ciências Biológicas, Av. José Moreira Sobrinho, s/no, Jequiezinho, CEP 45200-000 Jequié, BA. E-mail: plscarneiro@gmail.com, carlosmalhado@gmail.com, fazinhanery19@hotmail.com, polli bio@hotmail.com (2)Empresa Baiana de Desenvolvimento Agrícola, Praça Coronel João Borges, no 1, Centro, CEP 45200-000 Jequié, BA. E-mail: alcionejunior@bol.com.br, serafimdobode1@yahoo.com.br (3)Embrapa Recursos Genéticos e Biotecnologia, Parque Estação Biológica, Av. W5 Norte, Asa Norte, CEP 70770-900 Brasília, DF. E-mail: samuel@cenargen.embrapa.br
\end{abstract}

\begin{abstract}
Resumo - O objetivo deste trabalho foi comparar o desenvolvimento ponderal, por meio de curva de crescimento e pesos ajustados e avaliar a diversidade entre ovinos cruzados Dorper com as raças locais, com base em características de carcaça e morfológicas. Foram realizadas pesagens dos animais cruzados em intervalos de aproximadamente 15 dias e avaliados os efeitos de sexo, ano e grupo genético. Na estimativa dos parâmetros das curvas, foi utilizado o modelo não-linear Logístico e, a fim de avaliar o grau de similaridade entre os animais dos grupos genéticos, foram utilizadas variáveis canônicas. $\mathrm{O}$ sexo dos animais não influenciou o peso. Houve efeito de ano e grupo genético sobre o desenvolvimento dos animais. Conforme o modelo Logístico para a curva de crescimento, o grupo genético Dorper x Santa Inês apresentou maior velocidade de crescimento, estimado pelo peso à maturidade e taxa de maturação, seguido dos grupos genéticos Dorper x Morada Nova e Dorper x Rabo Largo. A análise de agrupamento ressaltou as diferenças entre os grupos genéticos e alocou os três cruzamentos em dois grupos, um formado pelos cruzamentos Dorper x Morada Nova e Dorper x Rabo Largo e o outro pelo cruzamento Dorper x Santa Inês.
\end{abstract}

Termos para indexação: Ovis aries, Morada Nova, Rabo Largo, Santa Inês, recursos genéticos animais.

\section{Growth rate and phenotypic diversity among crosses of Dorper ovines and local breeds}

\begin{abstract}
The goal of this work was to compare growth rate, by mean of growth and adjusted weight curves, and to analyze the diversity among crosses of Dorper ovines and local breeds, based on morphological and carcass features. Crossed animals were weighed in a 15-day-interval and the effects of sex, year and genetic group were analyzed. The non-linear Logistic model was used to estimate curve parameters and the degree of similarity among genetic groups was evaluated through canonic variables. Sex did not influence significantly on weight. There was a significant effect related to year and genetic group over animal's development. The estimates of growth curve based on parameters from the Logistic model indicated that the genetic group Dorper x Santa Inês presented a faster growth rate, as estimated by weight at maturity and maturity rate, followed by the genetic groups Dorper x Morada Nova and Dorper x Rabo Largo. Clustering analysis reinforced the differences among genetic groups, placing the three crosses in two groups, one comprising Dorper x Morada Nova and Dorper $\mathrm{x}$ Rabo Largo, and another including the cross Dorper x Santa Inês.
\end{abstract}

Index terms: Ovis aries, Morada Nova, Rabo Largo, Santa Inês, animal genetic resources.

\section{Introdução}

A Região Nordeste possui 8,7 milhões de ovinos, correspondendo a 57,8\% dos rebanhos do País. O Estado da Bahia concentra os maiores plantéis dessa espécie, com 2,9 milhões de ovinos (IBGE, 2004). Embora numericamente expressivo, esses rebanhos apresentam níveis acentuadamente reduzidos de desempenho. As raças ovinas deslanadas são adaptadas à Região Nordeste, entretanto apresentam baixos índices de produtividade (Sousa \& Leite, 2000), que estão intimamente relacionados à falta de caracterização de um sistema de produção adequado, de maneira a usar raças apropriadas para cada objetivo de produção. 
Em geral, raças localmente adaptadas ou simplesmente locais (FAO, 2001) do Nordeste brasileiro são adaptadas às condições edafoclimáticas dessa região, porém carecem de precocidade de acabamento e qualidade de carcaça (Barros et al., 2005).

A raça Dorper, originária da África do Sul, é um composto das raças Dorset com a Black Head Persian que, no Brasil, é denominada de Somalis Brasileira. Essa raça apresenta alta taxa de desenvolvimento e crescimento da carcaça com boa conformação (Souza \& Leite, 2000) e tem sido muito utilizada em cruzamento com ovelhas nativas deslanadas.

As curvas de crescimento de determinada raça podem auxiliar no estabelecimento de programas alimentares específicos e na definição da idade ótima de abate. De acordo com Freitas (2005), entre as várias aplicações das curvas de crescimento na produção animal, destacam-se: resumir em três ou quatro parâmetros, as características de produção, pois alguns parâmetros dos modelos não lineares utilizados possuem interpretabilidade biológica; avaliar o perfil de resposta de tratamento ao longo do tempo; estudar interações de respostas das subpopulações ou tratamentos com o tempo; identificar em uma população os animais mais pesados em idades mais jovens.

Segundo Cruz \& Regazzi (2001), o estudo de divergência - populações, tratamentos, genótipos entre outros - pode ser feito utilizando-se a técnica de variáveis canônicas, que consiste na transformação de um conjunto original de variáveis em variáveis padronizadas e não correlacionadas, com a característica de manter o princípio do processo de conglomeração com base na distância $\mathrm{D}^{2}$ de Mahalanobis. Esse novo conjunto de variáveis, em ordem de estimação, retém o máximo de informação em termos de variação total.

A análise de agrupamento tem por finalidade reunir objetos semelhantes, segundo suas características (variáveis), conforme algum critério de similaridade ou dissimilaridade, de tal forma que existam homogeneidade dentro do grupo e heterogeneidade entre grupos.

Uma das formas de se aumentar a capacidade produtiva dos rebanhos ovinos, com o propósito de atender às necessidades do mercado é o uso do cruzamento de raças especializadas com ovelhas nativas deslanadas. Este beneficia o uso da complementaridade entre raças, prática que favorece a conjugação das características desejáveis de cada raça, e a exploração da heterose. Entretanto, poucas informações estão disponíveis sobre a avaliação de cruzamentos entre diferentes raças de ovinos.

O objetivo deste trabalho foi comparar o desenvolvimento ponderal, por meio de curva de crescimento e pesos ajustados e avaliar a diversidade entre ovinos cruzados Dorper com as raças locais Morada Nova, Rabo Largo e Santa Inês, com base em características de carcaça e morfológicas.

\section{Material e Métodos}

O experimento foi conduzido na Estação Experimental de Jaguaquara, pertencente à Empresa Baiana de Desenvolvimento Agrícola S.A., localizada no Município de Jaguaquara, BA, microrregião administrativa de Jequié, BA, de 2003 a 2005. Foram avaliados os produtos $\mathrm{F}_{1}$, compreendendo borregos, machos e fêmeas, dos cruzamentos Dorper x Morada Nova (DMN); Dorper $x$ Rabo Largo (DRL) e Dorper x Santa Inês (DSI). A precipitação média foi de 589, 792 e $854 \mathrm{~mm}$ nos anos 2003, 2004 e 2005, respectivamente. Na Figura 1 é apresentada a variação mensal durante os anos.

O rebanho experimental foi composto por 69 matrizes pluríparas: 23 Santa Inês, 23 Morada Nova e 23 Rabo Largo, em condições de reprodução e devidamente selecionadas. Os reprodutores foram em número de dois, da raça Dorper, em condições de reprodução, utilizados de forma que cada reprodutor cobrisse $50 \%$ das matrizes de cada grupo racial.

As crias (Dorper x Morada Nova - DMN; Dorper x Rabo Largo - DRL; Dorper x Santa Inês - DSI) acompanharam as matrizes nas pastagens até os 84 dias, quando foram desmamadas. Os produtos $\mathrm{F}_{1}$, após o desmame, foram mantidos em sistema de produção semiintensivo, com pastejo durante o dia, em áreas cultivadas com capim Panicum maximum (Sempre-Verde) e

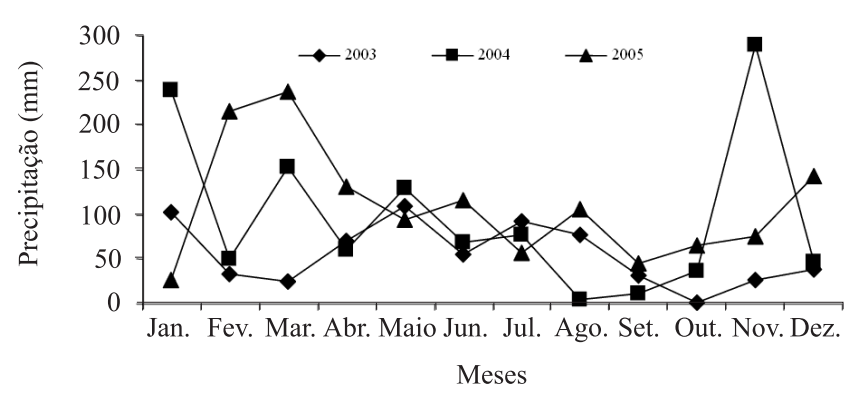

Figura 1. Médias mensais de precipitação pluvial no local do experimento. 
Brachiaria decumbens, com cobertura de 30 e $70 \%$, respectivamente, com sal mineral comercial à disposição, e recolhidos no final da tarde para o aprisco. Durante o inverno, período mais seco do ano, de junho a outubro, quando há decréscimo na qualidade da pastagem, foi oferecida suplementação com mistura múltipla comercial.

Foram realizadas pesagens do nascimento até os 150 dias de idade em intervalos de aproximadamente 15 dias. Os dados foram ajustados para pesos aos 30 dias de idade (P30: 73 observações), 60 (P60: 126), 90 (P90: 131), 120 (P120: 120) e 150 (P150: 74) e avaliados os efeitos de sexo, ano e grupo genético. A fim de comparar as médias estimadas pelo método dos quadrados mínimos, foi utilizado o teste t. As análises estatísticas foram realizadas utilizando-se o procedimento GLM do software SAS (2000).

Nas estimativas dos parâmetros das curvas, foi utilizado o modelo não-linear Logístico pelo procedimento NLIN do programa SAS (2000) com o método de Gauss Newton, cuja equação é dada por $\mathrm{y}_{\mathrm{t}}=\mathrm{A} /(1+\mathrm{b} \exp (-\mathrm{kt}))$, em que y representa o peso em $\mathrm{kg}$; $\mathrm{t}$, a idade em dias; A é o peso assintótico ou peso adulto; b, uma constante de integração; $k$ é a taxa de maturidade. As pesagens consideradas foram coletadas de 36 animais DMN, 61 animais DRL e 51 animais DSI.

Quanto às características de carcaça, foram utilizados 24 animais DMN, 23 DRL e 23 DSI e avaliadas as características peso da carcaça fria (PCF), grau de cobertura de gordura (GG), peso do pescoço (PESC), peso da meia carcaça esquerda (PMC), peso da paleta (Paleta), peso da costeleta (Costeleta) e peso do lombo (Lombo). As características morfológicas avaliadas foram altura da cernelha (Acer), altura dos costados (Acos), altura da garupa (Agar), largura do peito (Larp), largura da garupa (Larg), perímetro torácico (PTOR), comprimento do corpo-diagonal (CCD) e comprimento do corpo-dorsal (CCS) de 29 animais DMN, 31 DRL e 29 DSI.

O abate dos animais, para obtenção das características e carcaça, foi realizado após jejum de 16 horas de alimento sólido, com atordoamento de marreta e, em seguida, corte na artéria carótida e veia jugular para coleta, pesagem e descarte do sangue. Posteriormente, foi realizada a evisceração e obtenção da carcaça inteira do animal para retirada e pesagem dos cortes.

Os dados de carcaça e morfologia foram ajustados para os 120 dias de idade e as análises estatísticas foram realizadas utilizando-se o programa SAS (2000).
O modelo estatístico foi definido considerando o delineamento inteiramente casualizado com $\mathrm{i}$ cruzamentos e $r$ animais, em que foram medidas $t$ características:

$\mathrm{y}_{\mathrm{itr}}=\mu_{\mathrm{t}}+\mathrm{C}_{\mathrm{it}}+\mathrm{e}_{\mathrm{itr}}$, em que

$\sum_{\mathrm{i}=1}^{3} \mathrm{C}_{\mathrm{it}}=0, \forall \mathrm{t}, \mathrm{i}=1,2,3 ; \mathrm{r}=1,2, \ldots, \mathrm{j}$;

$\mathrm{y}_{\text {itr }}$ é o valor observado da característica t, do cruzamento $i$, no animal $\mathrm{r} ; \mu_{\mathrm{t}}$ é a média da característica t; $\mathrm{C}_{\mathrm{it}}$ é o efeito do cruzamento i na característica t; e $\mathrm{e}_{\text {itr }}$ é o erro aleatório atribuído à observação $\mathrm{y}_{\mathrm{itr}}$, em que os e ${ }^{\prime}{ }_{i r}=\left(e_{i P r}, \ldots, e_{i 3 r}\right)$ têm distribuição multinormal de dimensão três, igual ao número de características, com vetor nulo de médias e matriz de variâncias e covariâncias $\Sigma$, comum a todas as combinações i e r.

A função discriminante linear de Fisher (FDF) foi aplicada aos dados de observações, o que permitiu a redução do espaço p dimensional a um espaço unidimensional. A partir deste ponto, realizou-se ANOVA para o teste de hipótese de igualdade dos cruzamentos.

A fim de avaliar o grau de similaridade entre os animais das populações, foram utilizadas variáveis canônicas que possibilitam a identificação de grupos similares em gráficos de dispersão bi ou tridimensionais.

A importância relativa das variáveis canônicas foi medida pela percentagem de seus autovalores (variâncias) em relação ao total dos autovalores; a percentagem da variância total que elas explicam.

Nas análises de agrupamentos, adotou-se a distância generalizada $\mathrm{D}^{2}$ de Mahalanobis, sobre a qual empregouse o método de otimização de Tocher como método de agrupamento para a formação dos grupos similares (Cruz \& Carneiro, 2003). A distância generalizada $\mathrm{D}^{2}$ de Mahalanobis foi a medida utilizada para estabelecer o grau de divergência nos três grupos estudados, obtendose matrizes de dissimilaridade entre os animais dos três cruzamentos.

\section{Resultados e Discussão}

Quanto às características de desenvolvimento ponderal, observou-se que o efeito de sexo não influenciou $(p>0,05)$ o peso dos animais. Por sua vez, observou-se efeito de ano e grupo genético $(p<0,05)$ sobre o desenvolvimento dos animais.

A única característica em que não se observou diferença significativa para grupo genético foi o peso aos 30 (P30) dias de idade. Na Figura 2, visualiza-se a descrição das curvas de crescimentos para os três grupos genéticos. Observa-se que os cordeiros cruzados DSI apresentaram pesos iniciais próximos aos dois outros 
cruzamentos e passaram a ter maior velocidade de crescimento em idades mais avançadas.

Na Tabela 1 são apresentadas as médias ajustadas, pelo método dos quadrados mínimos, para os efeitos de sexo, ano e grupo genético. Quanto ao efeito de ano, não se observou tendência de melhor desempenho dos animais nas várias características. Quanto ao P90, observou-se que as médias dos anos 2003 e 2004 não diferiram entre si, apesar de serem os valores mais distantes. Isto provavelmente está relacionado à existência de heterogeneidade da variância na característica. Guedes et al. (2004) utilizaram ajuste ponderado das funções de crescimento em razão da constatação da heterogeneidade de variâncias nos dados relativos a peso-idade.

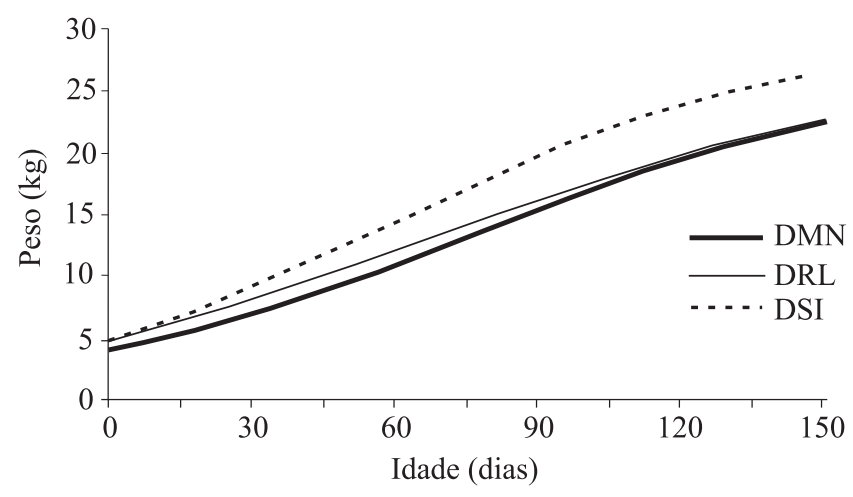

Figura 2. Estimativa do peso dos cruzamentos Dorper x Morada Nova (DMN), Dorper x Rabo Largo (DRL) e Dorper x Santa Inês (DSI) em função da idade, ajustado pelo modelo Logístico, até 150 dias de idade.
Com relação ao efeito de grupo genético, observouse que o cruzamento DSI apresentou desempenho superior aos cruzamentos DMN e DRL após os 30 dias de idade, e que não diferiram entre si. A raça Santa Inês tem sido muito utilizada em virtude do seu porte e da velocidade de crescimento. Do ponto de vista biológico, é um material genético novo e, de certa forma, ainda pouco conhecido, entretanto verifica-se algum tipo de seleção, mesmo que empírica, o que justifica seu maior desempenho em cruzamento quando comparado às raças Morada Nova e Rabo Largo.

Quesada et al. (2002) avaliaram o desempenho das raças Morada Nova (MN), Santa Inês (SI) e mestiços Texel x Morada Nova (T x MN). A raça SI e os mestiços apresentaram pesos maiores que a raça MN. Entretanto, os mestiços apresentaram maior intervalo de partos. Estes autores concluíram que há necessidade de novos estudos de caráter econômico para a raça Morada Nova, principalmente sobre o seu uso em sistemas de cruzamento.

Barros et al. (2004) observaram similaridade entre os grupos genéticos $1 / 2$ Somalis Brasileira x SRD e $1 / 2$ Santa Inês x SRD. Neste trabalho os pesos médios aos 56 e 112 dias foram 12,44 e 12,15 e 19,43 e 19,94 para os grupos genéticos $1 / 2$ Somalis Brasileira $x$ SRD e $1 / 2$ Santa Inês X SRD, respectivamente. Estes resultados são superiores aos observados neste estudo no que se refere aos cruzamentos DMN e DRL e inferiores, ao DSI.

Barros et al. (2005) avaliaram a eficiência bioeconômica de cordeiros $\mathrm{F}_{1}$ Dorper x Santa Inês para produção de carne, nas fases de cria e de acabamento.

Tabela 1. Médias (kg) estimadas pelos mínimos quadrados para sexo, ano de nascimento e grupo genético das características de peso (P) aos 30, 60, 90, 120 e 150 dias de idade em ovinos Dorper x Morada Nova (DMN), Dorper x Rabo Largo (DRL) e Dorper x Santa Inês (DSI) ${ }^{(1)}$.

\begin{tabular}{lccccc}
\hline Efeitos & P30 & P60 & P90 & P120 & P150 \\
\hline Sexo & & & & & \\
M & 7,71 & 11,99 & 15,74 & 19,49 & 23,73 \\
F & 7,63 & 11,39 & 14,57 & 18,00 & 21,41 \\
\hline Ano & & & & & \\
2003 & 7,02 & $10,28 \mathrm{a}$ & $14,22 \mathrm{a}$ & $18,90 \mathrm{~b}$ & $24,37 \mathrm{a}$ \\
2004 & - & $12,16 \mathrm{~b}$ & $16,56 \mathrm{a}$ & $20,09 \mathrm{a}$ & $23,43 \mathrm{a}$ \\
2005 & 8,32 & $12,63 \mathrm{~b}$ & $14,68 \mathrm{~b}$ & $17,24 \mathrm{~b}$ & $19,92 \mathrm{~b}$ \\
\hline Grupo genético & & & & & \\
DMN & 6,96 & $10,41 \mathrm{a}$ & $13,51 \mathrm{a}$ & $16,84 \mathrm{a}$ & $20,12 \mathrm{a}$ \\
DRL & 7,48 & $10,64 \mathrm{a}$ & $14,15 \mathrm{a}$ & $2,36 \mathrm{a}$ & $21,08 \mathrm{a}$ \\
DSI & 8,57 & $14,02 \mathrm{~b}$ & $17,81 \mathrm{~b}$ & $22,04 \mathrm{~b}$ & $26,51 \mathrm{~b}$ \\
\hline
\end{tabular}

${ }^{(1)}$ Médias seguidas de letras distintas diferem entre si $(\mathrm{p}<0,05)$ pelo teste $\mathrm{t}$. 
Realizaram a fase de produção das crias em caatinga nativa e a fase de acabamento em confinamento e comprovaram que o acabamento de cordeiros Dorper $\mathrm{x}$ Santa Inês, em confinamento, é viável economicamente.

As estimativas dos parâmetros do modelo Logístico (Tabela 2) indicaram que o grupo genético DSI apresentou maior potencial de crescimento, estimado pelo peso à maturidade (A) e maior taxa de maturação (k). Com relação à qualidade do ajuste, avaliada pelos coeficientes de determinação, foram semelhantes e adequados nos três cruzamentos avaliados, com valores acima de $98 \%$. Entre os grupos genéticos DMN e DRL, não foi observada diferença com relação ao potencial de crescimento (Figura 2).

O parâmetro A fornece uma estimativa do valor ou peso assintótico que não é, necessariamente, o maior peso que o animal atinge, mas sim o peso médio à maturidade, livre das variações sazonais (Fitzhugh Junior, 1976). Este parâmetro é mais representativo do peso adulto, quando as condições de ambiente forem mais constantes. Existem controvérsias sobre o tamanho adulto ótimo, que depende da espécie, raça, seleção praticada previamente, do sistema de manejo e das condições climáticas.

Apesar de não se observar o padrão clássico das curvas de crescimento, decorrente do abate precoce dos animais em análise, o ajuste do modelo não-linear Logístico é válido por permitir melhor interpretação do crescimento dos animais dos diferentes cruzamentos (Figura 2). Isso ocorre em razão de os parâmetros do modelo Logístico não-Linear condensarem informações importantes como precocidade e peso à maturidade, que não seriam possíveis de serem obtidas por um simples ajuste de regressão linear. No entanto, para uma estimativa precisa da curva de crescimento, é importante que

Tabela 2. Estimativas médias dos parâmetros A, $\mathrm{k}$ e b do modelo Logístico, no ajuste individual, e indicador de qualidade $\left(\mathrm{R}^{2}\right)$ nos três cruzamentos Dorper ${ }^{(1)}$.

\begin{tabular}{lrrr}
\hline Parâmetro & \multicolumn{1}{c}{ DMN } & \multicolumn{1}{c}{ DRL } & \multicolumn{1}{c}{ DSI } \\
\hline $\mathrm{A}$ & 27,1493 & 26,0857 & 28,6809 \\
$\mathrm{k}$ & 0,0223 & 0,0213 & 0,0267 \\
$\mathrm{~b}$ & 5,5894 & 4,1320 & 4,9236 \\
$\mathrm{R}^{2}$ & 98,49 & 98,12 & 98,09 \\
\hline
\end{tabular}

(1)DMN: Dorper x Morada Nova; DRL: Dorper x Rabo Largo; DSI: Dorper x Santa Inês; A é o peso assintótico ou peso adulto; b é a constante de integração; $k$ é a taxa de maturidade. os animais sejam controlados por um período de tempo maior.

Guedes et al. (2004) avaliaram curvas de crescimento de cordeiros das raças Santa Inês e Bergamácia e observaram comportamento praticamente linear, utilizando os modelos Logístico, Brody, Gompertz, Richards e von Bertalanffy.

A análise de variância (ANOVA) detectou diferenças significativas apenas quanto às características morfológicas ACER e CCD e quanto às características de carcaça Paleta e Costeleta. As funções lineares discriminantes de Fisher obtidas (FDF morfológicas = 0,17ACER - 0,36ACOS + 0,40AGAR + 0,199LARP - 0,22LARG - 0,066PTOR + $0,05 \mathrm{CCD}-0,023 \mathrm{CCS}$; FDF carcaça $=0,24 \mathrm{PCF}-1,67 \mathrm{GG}$ 0,708 PESC $-1,56 \mathrm{PMC}+13,60$ Paleta $+1,45$ Costeleta + 1,70 Lombo) apresentaram diferenças significativas $(\mathrm{p}<0,01)$ pelo teste $\mathrm{F}$ tanto para as características morfológicas quanto as de carcaça. Isto reflete uma das vantagens da análise multivariada (MANOVA) que fornece resultados da análise conjunta de todas as variáveis utilizadas, além de estimar a melhor combinação de variáveis que conduz ao valor máximo de $\mathrm{F}$.

Nas Tabelas 3 e 4 são apresentadas médias estimadas pelos mínimos quadrados nos três cruzamentos quanto às características morfológicas e de carcaça, respectivamente. Pode-se observar superioridade do cruzamento DSI em todas as características, exceto para GG, em que o cruzamento DRL foi superior, e para PTOR, em que DMN e DRL foram superiores. Na raça Rabo Largo é característico o acúmulo de gordura, especialmente na região da cauda, o que confere a estes animais grande adaptação às condições críticas do SemiÁrido.

O retorno econômico de dois sistemas de terminação de cordeiras $1 / 2$ Dorset Santa Inês, confinamento e pastagem mais a suplementação, foi avaliado com relação a características de carcaça por Santello et al. (2006). Estes autores concluíram que cordeiras 1/2 Dorset Santa $x$ Inês podem ser terminadas em confinamento ou em pastagem mais a suplementação, porém, considerandose a análise econômica, recomenda-se a terminação em pastagem mais a suplementação.

Pela análise de variáveis canônicas, observou-se que a primeira variável transformada foi suficiente para explicar aproximadamente $100 \%$ da variação total nos dados originais. Segundo Cruz \& Carneiro (2003), quando as primeiras variáveis explicam mais de $80 \%$ da variação total, é viável o estudo da divergência por meio das distâncias entre linhagens em gráficos de dispersão, cujas 
coordenadas são escores relativos às primeiras variáveis canônicas.

Na análise das medidas de dissimilaridade (Tabela 5), verificou-se que o cruzamento DSI apresentou-se mais distante dos cruzamentos DMN e DRL. O maior valor de dissimilaridade foi observado entre DSI e DRL (2,24: morfológicas). Já os cruzamentos mais próximos foram DMN e DRL (0,74: carcaça), com menor valor de dissimilaridade. Quando as distâncias do cruzamento Dorper com a raça Santa Inês (DSI) foram comparadas com os cruzamentos com as raças sob preservação Morada Nova (DMN) e Rabo Largo (DRL), observou-se que os cruzados DMN se aproximam mais do DSI (0,93 carcaça e 1,10 morfológicas) do que os cruzados DRL (1,76 carcaça e 2,4 morfológicas). O método de otimização de Tocher agrupou os três cruzamentos em dois grupos; o primeiro, formado por DMN e DRL e o segundo, por DSI (Figura 3).

Estes resultados podem refletir a formação da raça Santa Inês que, segundo Araújo \& Simplicio (2002), teve seu início a partir de gerações sucessivas de cruzamento entre a raça deslanada Morada Nova e raças lanadas ou semi-lanadas introduzidas no Nordeste, em especial, a Bergamácia.

A Santa Inês é a raça ovina de maior expansão no território nacional, visto que é encontrada em todo o Nordeste bem como em vários estados do Sudeste, Centro-Oeste e Norte do país. É de grande porte, apresenta boa capacidade de crescimento e boa produção de leite, o que lhe confere condições para criar bem, porém, apresenta, de maneira geral, baixa taxa de partos múltiplos (Barros et al., 2005). Silva (2002) avaliou o desempenho de ovinos Santa Inês, Somalis e mestiços Santa Inês x Crioulas, criados em pastagem nativa, na Região Semi-Árida do Nordeste do Brasil e observou que o potencial genético da raça Santa Inês contribuiu para que as crias Santa Inês e suas mestiças apresentassem melhor desempenho quando comparado às Somalis.

As raças Morada Nova e Rabo Largo, apesar dos menores valores para as características de carcaça avaliadas, apresentam vantagens que estão relacionadas, principalmente, à fertilidade e adaptação a ambientes muitas vezes inóspitos a outras raças. Silva \& Araújo (2000) avaliaram efeito de ambiente e genótipo, em características de reprodução e crescimento, em ovelhas crioulas, Santa Inês e cordeiros $F_{1}$ crioulos x Santa Inês, no Ceará. O grupo genético não exerceu efeito significativo sobre as variáveis de crescimento, porém, nas características reprodutivas, houve superioridade dos animais da raça crioula.

Assim, estudos que visem à avaliação, conservação e melhoramento dessas raças justificam-se, com animais puros ou cruzados. A conservação dessas raças está intimamente ligada à sua utilização e futura fonte de material genético capaz de melhorar a resistência de outras raças a condições desfavoráveis (Notter, 1999).

Tabela 3. Médias (cm) estimadas pelos mínimos quadrados para altura da cernelha (ACER), altura dos costados (ACOS), altura da garupa (AGAR), largura do peito (LARP), largura da garupa (LARG), perímetro torácico (PTOR), comprimento do corpodiagonal (CCD) e comprimento do corpo-dorsal (CCS).

\begin{tabular}{lcccccccc}
\hline Cruzamento $^{(1)}$ & ACER & ACOS & AGAR & LARP & LARG & PTOR & CCD & CCS \\
\hline DMN & 51,05 & 52,26 & 52,61 & 16,29 & 14,30 & 58,80 & 43,08 & 51,88 \\
DRL & 50,46 & 51,27 & 50,97 & 15,80 & 14,60 & 61,11 & 45,83 & 52,41 \\
DSI & 53,74 & 53,88 & 54,62 & 16,63 & 14,71 & 58,01 & 47,46 & 53,28 \\
\hline
\end{tabular}

${ }^{(1)}$ DMN: Dorper x Morada Nova; DRL: Dorper x Rabo Largo; DSI: Dorper x Santa Inês.

Tabela 4. Médias (kg) estimadas pelos mínimos quadrados para peso da carcaça fria (PCF), grau de cobertura de gordura (GG), peso do pescoço (PESC), peso da meia carcaça esquerda (PMC), peso da paleta, peso da costeleta e peso do lombo.

\begin{tabular}{lccccccc}
\hline Cruzamento $^{(1)}$ & PCF & GG & PESC & PMC & Paleta & Costeleta & Lombo \\
\hline DMN & 7,34 & 1,28 & 0,63 & 3,29 & 0,47 & 0,48 & 0,25 \\
DRL & 7,28 & 1,55 & 0,63 & 3,29 & 0,46 & 0,49 & 0,24 \\
DSI & 8,65 & 1,40 & 0,72 & 3,94 & 0,58 & 0,62 & 0,29 \\
\hline
\end{tabular}

${ }^{(1)}$ DMN: Dorper x Morada Nova; DRL: Dorper x Rabo Largo; DSI: Dorper x Santa Inês. 
Tabela 5. Distância generalizada de Mahalanobis $\left(D^{2}\right)$ entre pares de cruzamentos para características de carcaça, acima da diagonal, e morfológicas, abaixo da diagonal.

\begin{tabular}{lccc}
\hline Cruzamento $^{(1)}$ & DMN & DRL & DSI \\
\hline DMN & 0 & 0,74 & 0,93 \\
DRL & 1,10 & 0 & 1,76 \\
DSI & 1,41 & 2,24 & 0 \\
\hline
\end{tabular}

(1)DMN: Dorper x Morada Nova; DRL: Dorper x Rabo Largo; DSI: Dorper x Santa Inês.
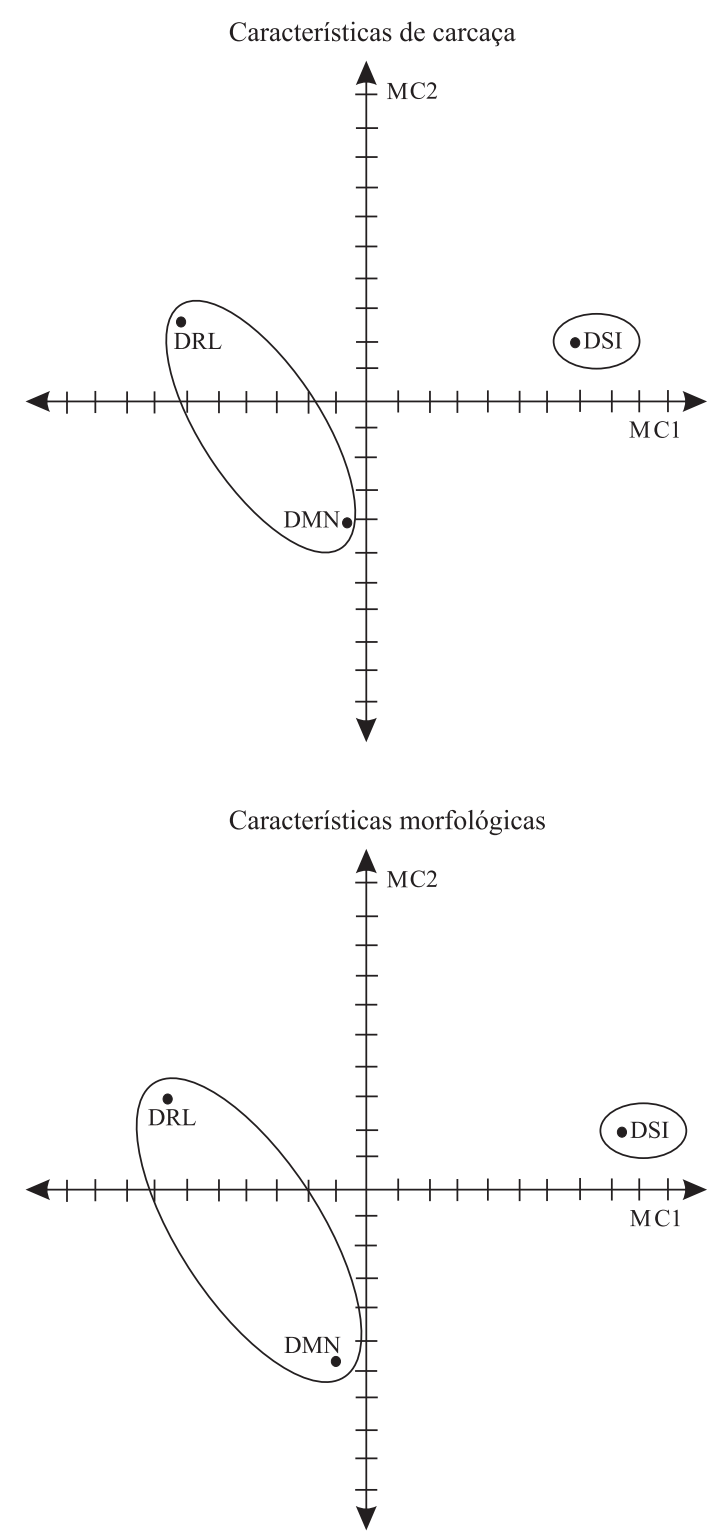

Figura 3. Dispersão gráfica dos três cruzamentos de ovinos Dorper x Morada Nova (DMN), Dorper x Rabo Largo (DRL) e Dorper x Santa Inês (DSI) com relação às duas primeiras variáveis canônicas, e agrupamento pelo método de Tocher quanto às características de carcaça e morfológicas.

\section{Conclusões}

1. O grupo genético Dorper x Santa Inês (DSI) apresenta maior velocidade de crescimento que os grupos Dorper x Morada Nova (DMN) e Dorper x Rabo Largo (DRL), após os 30 dias de idade.

2. Os produtos DSI são superiores aos DMN e DRL quanto às características morfológicas e de carcaça analisadas.

\section{Agradecimentos}

À Universidade Estadual do Sudoeste da Bahia, à Empresa Baiana de Desenvolvimento Agrícola, à Fapesb e ao CNPq, pelo apoio financeiro.

\section{Referências}

ARAÚJO, A.M.; SIMPLÍCIO, A.A. Melhoramento genético em caprinos e ovinos no Brasil: importância do padrão racial. In: SIMPÓSIO NACIONAL DE MELHORAMENTO ANIMAL, 2002, Campo Grande. Anais. Campo Grande, 2002. p.194-197.

BARROS, N.N.; VASCONCELOS, V.R.; LOBO, R.N.B. Características de crescimento de cordeiros de cordeiros $\mathrm{F}_{1}$ para abate no semi-árido do Nordeste do Brasil. Pesquisa Agropecuária Brasileira, v.39, p.809-814, 2004.

BARROS, N.N.; VASCONCELOS, V.R.; WANDER, A.E.; ARAÚJO, M.R.A. Eficiência bioeconômica de cordeiros $\mathrm{F}_{1}$ Dorper x Santa Inês para produção de carne. Pesquisa Agropecuária Brasileira, v.40, p.825-831, 2005.

CRUZ, C.D; CARNEIRO, P.C.S. Modelos biométricos aplicados ao melhoramento genético. Viçosa: UFV, 2003. v.2. 585p.

CRUZ, C.D.; REGAZZI, A.J. Modelos biométricos aplicados ao melhoramento genético. Viçosa: UFV, 2001. 390p.

FAO. Preparation of the first report on the State of the World's Animal Genetic Resources. Guidelines for the development of country reports. Animal Genetic Resources Information, n.30, 2001. 156p. (Anexo 2 - Working definitions for use in developing country reports and providing supporting data).

FITZHUGH, H.A. Analysis of growth curves and strategies for altering their shape. Journal of Animal Science, v.42, p.10361051, 1976.

FREITAS, A.R. Curvas de crescimento na produção animal. Revista Brasileira de Zootecnia, v.34, p.786-795, 2005.

GUEDES, M.H.P.; MUNIZ, J.A.; PEREZ, J.R.O.; SILVA, F.F.; AQUINO, L.H.; SANTOS, C.L. Estudo das curvas de crescimento de cordeiros das raças Santa Inês e Bergamácia considerando heterogeneidade de variâncias. Ciência Agrotécnica, Lavras, v.28, p.381-388, 2004.

IBGE - Pesquisa Pecuária Municipal. Rebanho ovino brasileiro: efetivo por unidade da federação 2004. Disponível em: http:// www.ibge.gov.br, Acesso em: 22 set. 2006. 
NOTTER D.R. The importance of genetic diversity in livestock populations of the future. Journal of Animal Science, v.77, 61-69, 1999.

QUESADA, M.; McMAnUS, C.; COUTO, F.A.D.A. Efeitos genéticos e fenotípicos sobre características de produção e reprodução de ovinos deslanados no distrito federal. Revista brasileira de Zootecnia, v.31, p.342-349, 2002. (Suplemento).

SANTELLO, G.A.; MACEDO, F.A.F.; MEXIA, A.A.; SAKAGUTI, E.S.; DIAS, F.J.; PEREIRA, M.F. Características de carcaça e análise do custo de sistemas de produção de cordeiras 1/2 Dorset Santa Inês. Revista Brasileira de Zootecnia, v.35, p.1852-1859, 2006. (Suplemento).
SAS - STATISTICAL ANALYSES SYSTEM. SAS/STAT user's guide. Cary: SAS Institute, 2000.

SILVA, F.L.R. Desempenho de ovinos deslanados e mestiços criados em pastagem nativa, na região semi-árida do nordeste do Brasil. Revista Científica de Produção Animal, v.4, p.7176, 2002.

SILVA, L.R.; ARAÚJO, A.M. Características de reprodução e de crescimento de ovinos mestiços Santa Inês no Ceará. Revista Brasileira de Zootecnia, v.29, p.1712-1720, 2000.

SOUSA, W.H.; LEITE, P.R.M. Ovinos de corte: a raça Dorper. João Pessoa: Emepa-PB, 2000. 76p.

Recebido em 28 de fevereiro de 2007 e aprovado em 19 de junho de 2007 